Article

\title{
Synthesis of a Pseudodisaccharide $\alpha-C$-Glycosidically Linked to an 8-Alkylated Guanine
}

\author{
Mu-Hua Huang ${ }^{1,2, *}$, Jan Duchek ${ }^{2}$ and Andrea Vasella ${ }^{2}$ \\ 1 School of Materials Science and Engineering, Beijing Institute of Technology, Beijing 100081, China \\ 2 Laboratorium für Organische Chemie, Departement Chemie und Angewandte Biowissenschaften, \\ ETH Zürich, HCI, Zürich CH-8093, Switzerland
}

* Author to whom correspondence should be addressed; E-Mail: mhhuang@bit.edu.cn; Tel./Fax: +86-10-6891-1608.

Received: 25 February 2013; in revised form: 21 March 2013 / Accepted: 21 March 2013 / Published: 2 April 2013

\begin{abstract}
The synthesis of stable guanofosfocin analogues has attracted considerable attention in the past 15 years. Several guanofosfocin analogues mimicking the three constitutional elements of mannose, ribose, and guanine were designed and synthesized. Interest in ether-linked pseudodisaccharides and 8-alkylated guanines is increasing, due to their potential applications in life science. In this article, a novel guanofosfocin analogue $\mathbf{6}$, an ether-linked pseudodisaccharide connected $\alpha$ - $C$-glycosidically to an 8 -alkylated guanine, was synthesized in a 10-longest linear step sequence from known diol 13, resulting in an overall yield of $26 \%$. The key steps involve the ring-opening of cyclic sulfate $\mathbf{8}$ by alkoxide generated from 7 and a reductive cyclization of 4- $\mathrm{N}$-acyl-2,4-diamino-5nitrosopyrimidine 19 to form compound 6.
\end{abstract}

Keywords: guanofosfocin; 8-alkylated guanine; ether-linked pseudodisaccharide; analogues; $\alpha$ - $C$-glycoside; nitrosopyrimidine

\section{Introduction}

Stable carbohydrate mimics are used widely to study the biological functions of oligo- and polysaccharides [1-3]. Well-known examples include $C$-glycosides [4], carbasugars [5] and thiooligosaccharides [6]. 
Guanofosfocin B (1, Figure 1) is one of the three guanofosfocins which were isolated in 1996 by Nippon Roche [7] from the fermentation broth of Streptmyces sp. AB 2570 and Trichoderma sp. FD 5372. Guanofosfocins are of interest as strong inhibitors of chitin synthases $\left(\mathrm{IC}_{50}: 1-10 \mathrm{nM}\right)$. Detailed investigations of their biological activity were, however, hampered by their rapid decomposition. The instability, not surprising considering the two activated acetal moieties found in $\mathbf{1}$, was addressed by several groups by synthesizing stable open chain analogues of $\mathbf{1}$ while potentially retaining the promising biological properties. Sugimura et al. designed several analogues such as 2 (Figure 1) that maintained the alkoxy substituent attached to the $C(8)$ of guanine [8-10]. Later on, they replaced the mannosyl moiety by a carba-mannosyl unit, and synthesized analogue 3 (Figure 1) [11]. Vasella et al. aimed at $C$-mannosides, replacing the anomeric oxygen by a methylene group, and prepared analogues 4 [12] and 5 [13] (Figure 1). We also considered the guanofosfocin analogue 6 of interest, in analogy to other, stable ether-linked pseudo-disaccharides [14-16] and report a synthesis of this ether-linked pseudo-disaccharide containing an 8-alkylated guanine.

Figure 1. Compounds prepared on the way to stable analogues of guanofosfocins.

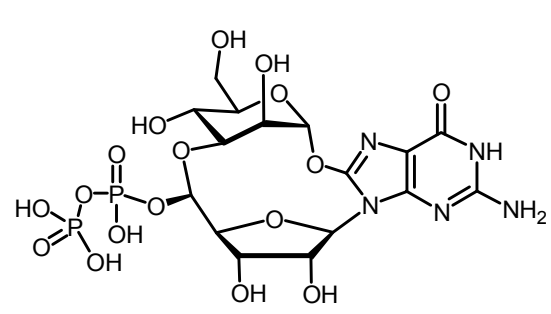

Guanofosfocin B (1)

Isolated in 1996

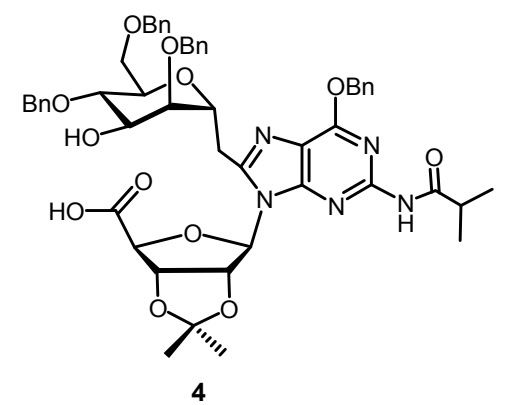

by Vasella et al. in 2004

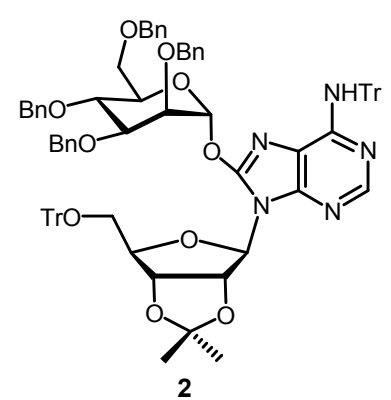

by Sugimura et al. in 1998

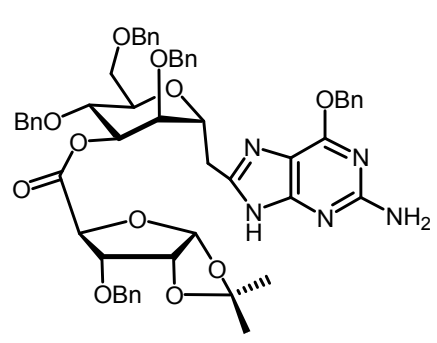

5

by Vasella et al. in 2011

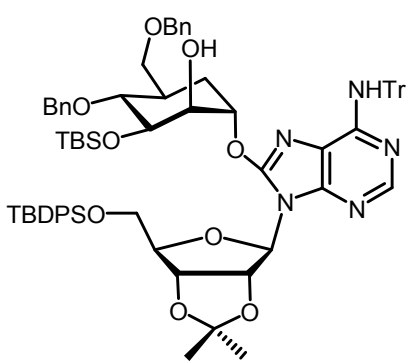

3

Sugimura et al. in 2007

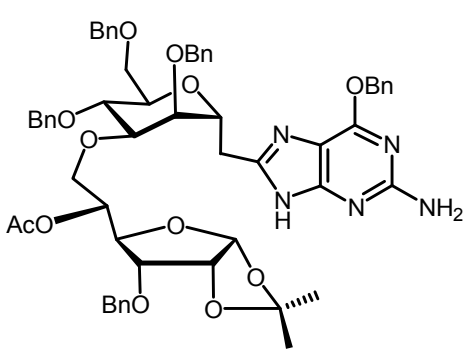

6

This work

\section{Results and Discussion}

The structure of $\mathbf{6}$ is characterized by an ether-linked pseudo-disaccharide with the $\alpha$ - $C$-mannopyranosyl unit linked to $\mathrm{C}(8)$ of a guanine via a methylene group. Accordingly, we had to incorporate a methylene group between the guanyl and mannosyl moieties and install the ether bond between the secondary $\mathrm{C}(3)-\mathrm{OH}$ group of mannose and the $\mathrm{C}(6)-\mathrm{OH}$ group of an allofuranose. Retrosynthetically (Scheme 1), the ether bond could be formed by ring-opening cyclic sulfate 8 by the alkoxy anion corresponding to alcohol 7 [17], and the 8-substituted guanine could be formed by regioselective 4- $\mathrm{N}$-acylation of 2,4-diamino-5-nitrosopyrimidine 9 , followed by reductive cyclization, using a procedure developed by Vasella et al. [18]. 
Scheme 1. Retrosynthesis of pseudodisaccharide-guanine hybrid 6.

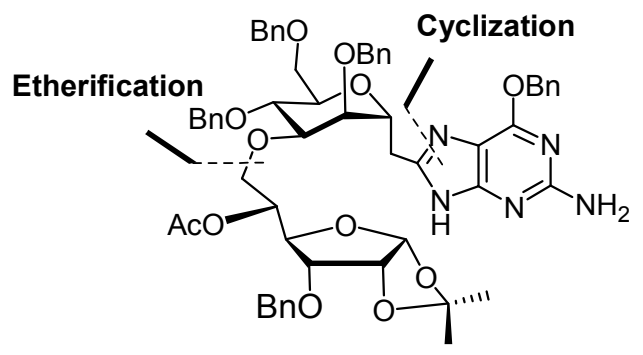

6

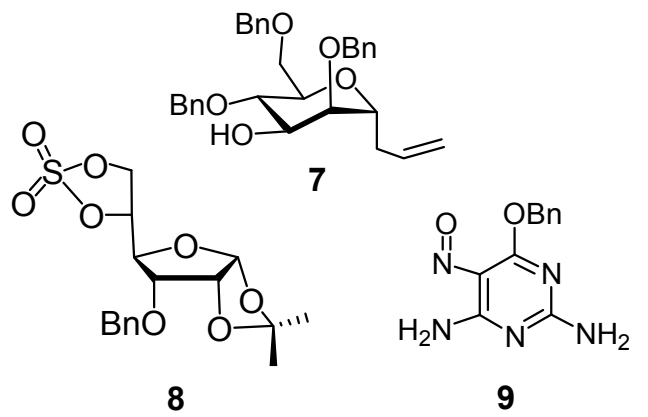

8

Our synthesis started with the preparation of the protected $\alpha$-allofuranose-diol 13, following known procedures as outlined in Scheme 2 [19-22]. The secondary alcohol 7 was synthesized from commercially available methyl $\alpha$-D-mannoside in $36 \%$ overall yield using the three-step sequence reported by Vasella et al. [13]. Epoxide formation from vicinal diol 13 under Mitsunobu conditions [23] yielded $66 \%$ of 14 . With epoxide 14 in hand, we carried out the oxirane ring-opening by the secondary alkoxide of 7 to get the pseudo-disaccharide 15 in 39\% yield, besides 49\% of recovered epoxide 14 .

Scheme 2. Synthesis of ether-linked pseudodisaccharide 15.

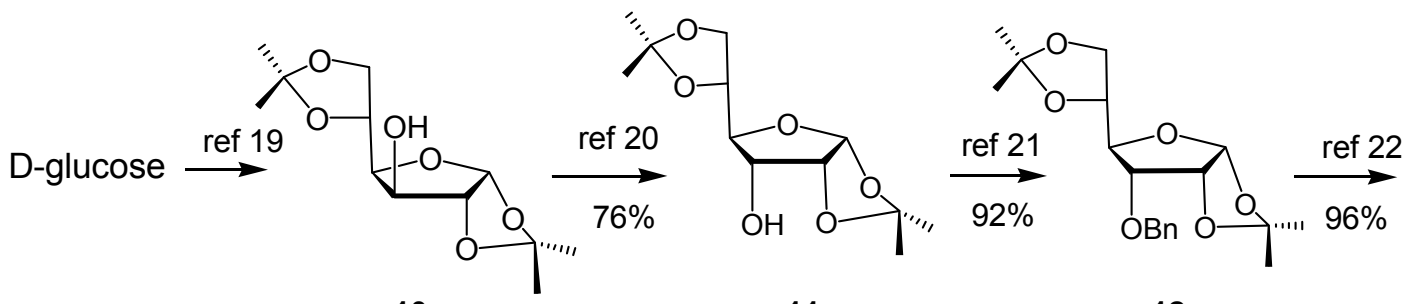

10

11
12

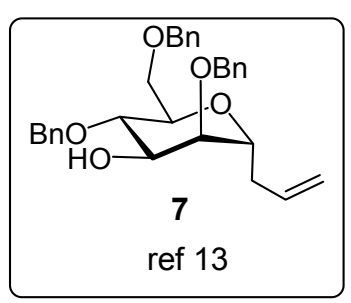

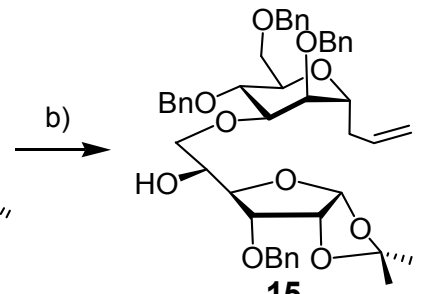

15
13

14

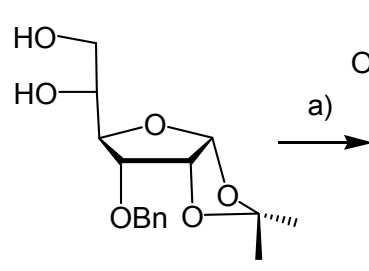

Reagents and conditions:

(a) $\mathrm{Ph}_{3} \mathrm{P}$, DIAD, toluene, 66\%; (b) 7, NaH, DMF, 39\%.

To improve the yield of the etherification, we prepared the cyclic sulfate $\mathbf{8}$ (Scheme 3 ). It was obtained in $69 \%$ from diol 13 by treatment with thionyl chloride and subsequent oxidation with $\mathrm{NaIO}_{4}$ in the presence of catalytic $\mathrm{Ru}(\mathrm{II}) \mathrm{Cl}_{2} \cdot \mathrm{xH}_{2} \mathrm{O}$ [24]. The cyclic sulfate 8 was a colorless solid that darkened upon storage, even in the refrigerator $\left(0-5{ }^{\circ} \mathrm{C}\right)$ and so was used immediately. The reaction between the cyclic sulfate 8 and the oxyanion derived from alcohol 7 in HMPA/THF took place smoothly to furnish the ether-linked pseudo-disaccharide 15 in a yield of $86 \%$ upon acidic aqueous work-up. The polar solvent proved crucial for the high yield. Alcohol 15 was acetylated and converted to the carboxylic acid 18 in a yield of $88 \%$ by dihydroxylation of 16 by $\mathrm{OsO}_{4} / \mathrm{NMO}$, cleavage of the resulting diol by $\mathrm{NaIO}_{4}$, and oxidation of the resulting aldehyde by $\mathrm{NaH}_{2} \mathrm{PO}_{4} / \mathrm{NaClO}_{2}$. Treatment of acid 18 with oxalyl chloride in the presence of catalytic DMF furnished the acid chloride. Though the 
reaction was carried out for $1 \mathrm{~h}$, the conversion was completed within $5 \mathrm{~min}$, as observed by monitoring the reaction mixture by IR spectroscopy. The acid chloride was stable enough to allow routine characterization (IR, ${ }^{1} \mathrm{H}-\mathrm{NMR}$ and ${ }^{13} \mathrm{C}-\mathrm{NMR}$ ). It reacted with 6-(benzyloxy)-5-nitrosopyrimidine-2,4diamine (9) to afford amide 19, accompanied by a color change from purple to blue-green. As purification of amide 19 by chromatography led to yields below $50 \%$, presumably caused by a strong absorption of amide 19 on silica gel, the crude amide 19 was treated directly with triphenylphosphine in xylene under reflux to furnish guanine $\mathbf{6}$ in an overall yield of 50\% from 18 (Scheme 3). The reductive cyclisation was accompanied by a color change from blue-green to brown.

Scheme 3. Synthesis of pseudodisaccharide-guanine hybrid 6 from cyclic sulfate 8.
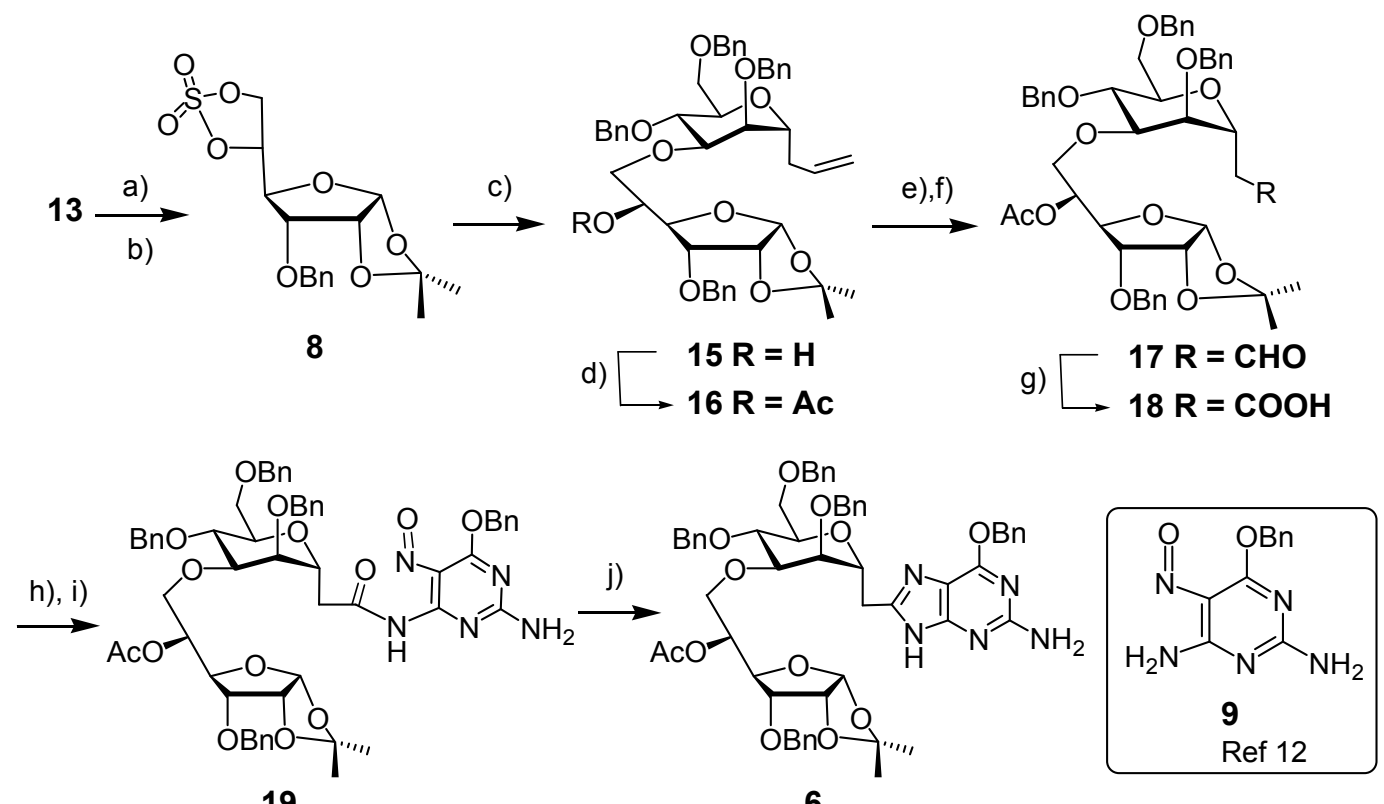

19

6

Reagents and conditions: (a) $\mathrm{SOCl}_{2}$, Pyr., $\mathrm{CH}_{2} \mathrm{Cl}_{2}$; (b) $\mathrm{RuCl}_{2} \cdot \mathrm{xH}_{2} \mathrm{O}, \mathrm{NaIO}_{4}, 69 \%$ for 2 steps; (c) 7, $\mathrm{NaH}$, HMPA/THF, 86\%; (d) $\mathrm{Ac}_{2} \mathrm{O}$, DMAP, $\mathrm{Et}_{3} \mathrm{~N}$; (e) $\mathrm{OsO}_{4}$, NMO; (f) $\mathrm{NaIO}_{4}$; (g) $\mathrm{NaH}_{2} \mathrm{PO}_{4}, \mathrm{NaClO}_{2}, 88 \%$ for 4 steps; (h) $(\mathrm{COCl})_{2}$, DMF(cat.), $\mathrm{CH}_{2} \mathrm{Cl}_{2}$; (i) 9, Pyr., THF; (j) $\mathrm{Ph}_{3} \mathrm{P}$, xylene, reflux; $50 \%$ for 3 steps.

With guanine $\mathbf{6}$ in hand, we performed a few scouting reactions to test its macrocyclization reactions via direct intra-molecular $N$-glycosylation. The first results showed that acetonide in 6 was not a good glycosyl donor for $N$-glycosylation. We then hydrolysed 6 to its corresponding vicinal diol. The macrocyclization is under investigation and the results will be reported in due course.

\section{Experimental}

\section{General}

Commercially available reagents were used without further purification. Water-free solvents were dried: THF was distilled from $\mathrm{Na}$ /benzophenone; toluene from $\mathrm{Na} ; \mathrm{CH}_{2} \mathrm{Cl}_{2}, \mathrm{MeCN}, \mathrm{MeOH}$, pyridine, and triethylamine from $\mathrm{CaH}_{2}$; acetone, and chloroform were dried over $4 \AA$ molecular sieves. Technical solvents were distilled: AcOEt, $\mathrm{CH}_{2} \mathrm{Cl}_{2}$ from $\mathrm{K}_{2} \mathrm{CO}_{3}$; $\mathrm{Et}_{2} \mathrm{O}$ from $\mathrm{FeSO}_{4} \cdot 7 \mathrm{H}_{2} \mathrm{O}$; cyclohexane, hexane, $\mathrm{MeOH}$, and toluene without any other additive. The reactions were carried out in oven-dried glassware, under an $\mathrm{N}_{2}$ or Ar atmosphere, unless stated otherwise. Qualitative TLC: precoated silica-gel 
plates (Merck silica gel $60 \mathrm{~F}_{254}$ ); detection by heating with 'mostain' (400 mL of 10\% $\mathrm{H}_{2} \mathrm{SO}_{4}$ soln., $20 \mathrm{~g}$ of $\left(\mathrm{NH}_{4}\right)_{6} \mathrm{Mo}_{7} \mathrm{O}_{24} \cdot 4 \mathrm{H}_{2} \mathrm{O}, 0.4 \mathrm{~g}$ of $\left.\mathrm{Ce}\left(\mathrm{SO}_{4}\right)_{2} \cdot 4 \mathrm{H}_{2} \mathrm{O}\right)$, or by UV. FCC (flash column chromatography): silica gel Fluka $60(0.04-0.063 \mathrm{~mm})$ or Merck silica gel $60(0.063-0.200 \mathrm{~mm})$ under slightly elevated pressure (0.1-0.4 bar). Melting points were measured on a Büchi B-540 melting point apparatus using open glass capillaries and are uncorrected. Optical rotations were measured with a PerkinElmer digital polarimeter: $1-\mathrm{dm}$ cell at $25{ }^{\circ} \mathrm{C}, 589 \mathrm{~nm}$, concentration (c) in g/100 mL. Infrared spectroscopy (IR) were recorded on a Perkin Elmer Spectrum RX-I FT-IR: ca. $2 \%$ soln. in $\mathrm{CHCl}_{3}$; absorptions in cm ${ }^{-1}$. NMR-spectra were recorded on Bruker magnetic resonance spectrometer $\left({ }^{1} \mathrm{H}\right.$ at $300 \mathrm{MHz},{ }^{13} \mathrm{C}$ at $\left.75 \mathrm{MHz}\right)$ : chemical shifts $\delta$ in ppm relative to a residual undeuterated solvent peak. MS spectra were recorded on an IONSPEC Ultima ESI-FT-ICR spectrometer at $4.7 \mathrm{~T}$.

3-O-Benzyl-1,2-O-isopropylidene- $\alpha$-D-allofuranose, 5,6-cyclic sulfate (8). A solution of thionyl chloride $(0.11 \mathrm{~mL}, 1.5 \mathrm{mmol})$ in $\mathrm{CH}_{2} \mathrm{Cl}_{2}(0.85 \mathrm{~mL})$ was added dropwise to an ice-cooled solution of diol 13 (230 mg, $0.74 \mathrm{mmol})$ in $\mathrm{CH}_{2} \mathrm{Cl}_{2}(5 \mathrm{~mL})$ and pyridine $(0.24 \mathrm{~mL}, 3 \mathrm{mmol})$. The mixture was stirred for $5 \mathrm{~min}$, when TLC revealed the disappearance of starting material. The mixture was diluted with $\mathrm{CH}_{2} \mathrm{Cl}_{2}$ and washed with water. The combined aqueous layers were extracted with $\mathrm{CH}_{2} \mathrm{Cl}_{2}$. The combined organic layers were dried $\left(\mathrm{Na}_{2} \mathrm{SO}_{4}\right)$ and concentrated. The residue was dissolved in $\mathrm{CH}_{2} \mathrm{Cl}_{2} / \mathrm{MeCN} / \mathrm{H}_{2} \mathrm{O}$ (2/2/3), to which was added $\mathrm{NaIO}_{4}(320 \mathrm{mg}, 1.5 \mathrm{mmol})$ followed by $\mathrm{Ru}(\mathrm{II}) \mathrm{Cl}_{2} \cdot \mathrm{xH}_{2} \mathrm{O}(10 \mathrm{mg})$. After $10 \mathrm{~min}$, the mixture was diluted with $\mathrm{CH}_{2} \mathrm{Cl}_{2}$, the organic layer was separated, the water layer was extracted with $\mathrm{CH}_{2} \mathrm{Cl}_{2}$. The combined organic layers were dried $\left(\mathrm{Na}_{2} \mathrm{SO}_{4}\right)$ and concentrated in vacuo. The residue was purified by Flash Column Chromatography (FCC, EtOAc/cyclohexane, $1 / 3 \rightarrow 1 / 1)$ to afford the cyclic sulfate 8 as a white solid $(191 \mathrm{mg}, 69 \%$ ). m.p. $120-122{ }^{\circ} \mathrm{C}$ (dec.) (EtOAc/hexane). ${ }^{1} \mathrm{H}-\mathrm{NMR}\left(\mathrm{CDCl}_{3}, 300 \mathrm{MHz}\right): \delta(\mathrm{ppm}) 7.40-7.37(\mathrm{~m}, 5 \mathrm{H})$, $5.74(\mathrm{~d}, 1 \mathrm{H}, J=3.3 \mathrm{~Hz}), 5.17(\mathrm{dt}, 1 \mathrm{H}, J=2.4,7.2 \mathrm{~Hz}), 4.79(\mathrm{dd}, 1 \mathrm{H}, J=7.8,9.0 \mathrm{~Hz}), 4.75(\mathrm{~d}, 1 \mathrm{H}$, $J=12.0 \mathrm{~Hz}), 4.62(\mathrm{~d}, 1 \mathrm{H}, J=12.0 \mathrm{~Hz}), 4.60(\mathrm{~d}, 1 \mathrm{H}, J=6.9 \mathrm{~Hz}), 4.57(\mathrm{dd}, 1 \mathrm{H}, J=1.2,5.4 \mathrm{~Hz}), 4.23$ $(\mathrm{dd}, 1 \mathrm{H}, J=2.4,9.0 \mathrm{~Hz}), 3.99(\mathrm{dd}, 1 \mathrm{H}, J=4.5,9.0 \mathrm{~Hz}), 1.59(\mathrm{~s}, 3 \mathrm{H}), 1.36(\mathrm{~s}, 3 \mathrm{H}) .{ }^{13} \mathrm{C}-\mathrm{NMR}$ $\left(\mathrm{CDCl}_{3}, 75 \mathrm{MHz}\right): \delta$ (ppm) 136.51, 128.53, 128.36, 113.53, 103.95, 79.99, 77.13, 77.05, 76.41, 72.59, 67.86, 26.90, 26.46. HR-MS(ESI), $m / z$ 395.07695 [M+Na] ${ }^{+}\left(\mathrm{C}_{16} \mathrm{H}_{20} \mathrm{NaO}_{8} \mathrm{~S}^{+}\right.$, required 395.07711).

5,6-Anhydro-3-O-benzyl1,2-O-isopropylidene- $\alpha$-D-allofuranose (14). To a solution of diol 13 (305 mg, $0.98 \mathrm{mmol})$ and triphenylphosphine $(310 \mathrm{mg}, 1.18 \mathrm{mmol})$ in dry toluene $(6 \mathrm{~mL})$ was added diisopropyl azodicarboxylate $(0.25 \mathrm{~mL}, 94 \%$ pure, $1.18 \mathrm{mmol})$ dropwise at room temperature. The mixture was stirred under reflux overnight. The solvent was removed in vacuo, the residue was purified by FCC (EtOAc/cyclohexane, 1/3) to give epoxide 14 as a colorless oil (189 mg, 66\%). IR (Film, $\left.\mathrm{cm}^{-1}\right): 3021$ (s), $2934(\mathrm{w}), 2870(\mathrm{w}), 1751(\mathrm{w}), 1455(\mathrm{w}), 1384(\mathrm{w}), 1375(\mathrm{w}), 1315(\mathrm{w}), 1254(\mathrm{w}), 1163(\mathrm{w}), 1131$ (m), 1102 (s), 1022 (s). ${ }^{1} \mathrm{H}-\mathrm{NMR}\left(\mathrm{CDCl}_{3}, 300 \mathrm{MHz}\right): \delta(\mathrm{ppm})$ 7.40-7.29 (m, $\left.5 \mathrm{H}\right), 5.75(\mathrm{~d}, 1 \mathrm{H}$, $J=3.9 \mathrm{~Hz}), 4.75(\mathrm{~d}, 1 \mathrm{H}, J=11.4 \mathrm{~Hz}), 4.59-4.55(\mathrm{~m}, 2 \mathrm{H}), 4.21(\mathrm{dd}, 1 \mathrm{H}, J=3.0,9.0 \mathrm{~Hz}), 3.66$ (dd, 1 $\mathrm{H}, J=4.2,8.7 \mathrm{~Hz}), 3.19(\mathrm{dd}, 1 \mathrm{H}, J=3.0,7.2 \mathrm{~Hz}), 2.81-2.73(\mathrm{~m}, 2 \mathrm{H}), 1.59(\mathrm{~s}, 3 \mathrm{H}), 1.36(\mathrm{~s}, 3 \mathrm{H})$. ${ }^{13} \mathrm{C}-\mathrm{NMR}\left(\mathrm{CDCl}_{3}, 75 \mathrm{MHz}\right): \delta(\mathrm{ppm}) 137.26,128.57,128.20,128.16,113.12,104.21,77.73,77.57$, $77.54,72.02,50.70,44.45,26.87,26.60 .[\alpha]_{\mathrm{D}}^{25}+85.92^{\circ}\left(c 1.05\right.$ in $\left.\mathrm{CHCl}_{3}\right)$. 
2,6-Anhydro-1,3,5-tri-O-benzyl-4-O-(3-O-benzyl-1,2-O-isopropylidene-6-deoxy- $\alpha$-D-allofuranos-6-yl)7,8,9-trideoxy-D-glycero-D-mannonon-8-enitol (15). To a mixture of $\mathrm{NaH}(60 \%$ in oil, $93 \mathrm{mg}, 2.32 \mathrm{mmol})$ in HMPA $(3 \mathrm{~mL})$ and THF $(1 \mathrm{~mL})$ was added alcohol $7(1.10 \mathrm{~g}, 2.32 \mathrm{mmol})$ in THF $(15 \mathrm{~mL})$. The resulting slightly yellow solution was stirred at room temperature for $20 \mathrm{~min}$, and then treated with cyclic sulfate $8(720 \mathrm{mg}, 1.93 \mathrm{mmol})$ in THF $(5 \mathrm{~mL})$. The resulting mixture was stirred at room temperature overnight when TLC revealed the disappearance of the cyclic sulfate. The reaction mixture was treated with $\mathrm{H}_{2} \mathrm{SO}_{4} / \mathrm{THF} / \mathrm{H}_{2} \mathrm{O}(1 / 100 / 0.3,3 \mathrm{~mL})$, stirred for $1 \mathrm{~h}$, treated with saturated aqueous $\mathrm{NaHCO}_{3}$ solution, and extracted with EtOAc. The combined organic layers were dried $\left(\mathrm{MgSO}_{4}\right)$, concentrated, and the residue purified by FCC (EtOAc/cyclohexane, 1/2 $\left.\rightarrow 1 / 1\right)$ to afford 15 as a colorless oil (1.28 g, 86\%). IR (Film, cm $\left.{ }^{-1}\right)$ : 3475 (w), 3067 (w), 3032 (w), 3009 (m), 2928 (m), 2872 (w), 1732 (w), 1672 (w), 1496 (w), 1454 (w), 1374 (w), 1313 (w), 1224 (m), 1094 (s), 1027 (s). ${ }^{1} \mathrm{H}-\mathrm{NMR}\left(\mathrm{CDCl}_{3}, 300 \mathrm{MHz}\right): \delta(\mathrm{ppm}) 7.34-7.23(\mathrm{~m}, 20 \mathrm{H}), 5.84-5.70(\mathrm{~m}, 1 \mathrm{H}), 5.72(\mathrm{~d}, 1 \mathrm{H}, J=3.3 \mathrm{~Hz})$, 5.08-5.01 (m, $2 \mathrm{H}), 5.72-4.48(\mathrm{~m}, 9 \mathrm{H}), 4.05-3.91(\mathrm{~m}, 4 \mathrm{H}), 3.86-3.48(\mathrm{~m}, 8 \mathrm{H}), 2.88$ (d, $1 \mathrm{H}, J=2.4 \mathrm{~Hz})$, 2.38-2.25 (m, $2 \mathrm{H}), 1.57$ (s, $3 \mathrm{H}), 1.35$ (s, $3 \mathrm{H}) .{ }^{13} \mathrm{C}-\mathrm{NMR}\left(\mathrm{CDCl}_{3}, 75 \mathrm{MHz}\right): \delta(\mathrm{ppm}) 138.22,137.94$, $137.90,137.40,134.16,128.29,128.19,127.94,127.87,127.64,127.39,117.17,112.86,104.03$, 78.66, 78.13, 77.71, 77.60, 75.14, 74.94, 73.86, 73.55, 73.29, 72.28, 72.13, 71.81, 71.10, 70.29, 68.96, 34.61, 26.93, 26.67. $\left([\alpha]_{\mathrm{D}}^{25}+45.45^{\circ}\right.$ (c 1.00 in $\mathrm{CHCl}_{3}$ ). HR-MS (ESI), $m / z 789.36095[\mathrm{M}+\mathrm{Na}]^{+}$ $\left(\mathrm{C}_{46} \mathrm{H}_{54} \mathrm{NaO}_{10}{ }^{+}\right.$, required 789.36092).

2,6-Anhydro-1,3,5-tri-O-benzyl-4-O-(5-O-acetyl-3-O-benzyl-1,2-O-isopropylidene-6-deoxy- $\alpha$-D-allofuranos6-yl)-7,8,9-trideoxy-D-glycero-D-mannonon-8-enitol (16). Triethylamine $(0.32 \mathrm{~mL}, 2.29 \mathrm{mmol})$ and DMAP $(61 \mathrm{mg}, 0.50 \mathrm{mmol})$ in dry $\mathrm{CH}_{2} \mathrm{Cl}_{2}(2 \mathrm{~mL})$, were added dropwise at $0{ }^{\circ} \mathrm{C}$ to a solution of alcohol $15(0.88 \mathrm{~g}, 1.15 \mathrm{mmol})$ and acetic anhydride $(0.21 \mathrm{~mL}, 2.29 \mathrm{mmol})$ in dry $\mathrm{CH}_{2} \mathrm{Cl}_{2}(3 \mathrm{~mL})$. The resulting mixture was stirred at room temperature overnight. The reaction was quenched by adding water $(1 \mathrm{~mL})$, and the solvent was removed in vacuo. A solution of the residue in EtOAc $(20 \mathrm{~mL})$ was washed with water and $0.1 \mathrm{~N} \mathrm{HCl}$. The aqueous layer was extracted with EtOAc, the combined organic layers were dried $\left(\mathrm{MgSO}_{4}\right)$ and concentrated to give the acetate 16 as a slightly yellow oil $(0.94 \mathrm{~g}$, 100\%). IR ( $\mathrm{cm}^{-1}, \mathrm{CHCl}_{3}$ ): 3067 (w), 3032 (w), 3010 (m), 2928 (w), 1738 (s), 1642 (w), 1496 (w), 1374 (m), 1374 (m), 1307 (w), 1240 (s), 1093 (s), 1027 (s). ${ }^{1} \mathrm{H}-\mathrm{NMR}\left(\mathrm{CDCl}_{3}, 300 \mathrm{MHz}\right)$ : $\delta$ (ppm) 7.34-7.26 (m, 20 H), 5.85-5.73 (m, $1 \mathrm{H}), 5.64$ (d, $1 \mathrm{H}, J=3.7 \mathrm{~Hz}), 5.31$ (ddd, $1 \mathrm{H}, J=4.8,3.0,6.6 \mathrm{~Hz}$ ), 5.11-5.04 (m, 2 H), $4.73(\mathrm{~d}, 1 \mathrm{H}, J=11.3 \mathrm{~Hz}), 4.67$ (d, $1 \mathrm{H}, J=11.2 \mathrm{~Hz}), 4.61-4.40$ (m, $7 \mathrm{H}), 4.12$ $(\mathrm{dd}, 1 \mathrm{H}, J=5.3,8.6 \mathrm{~Hz}), 4.05-3.98(\mathrm{~m}, 1 \mathrm{H}), 3.90(\mathrm{dd}, 1 \mathrm{H}, J=4.3,8.9 \mathrm{~Hz}), 3.83-3.62(\mathrm{~m}, 8 \mathrm{H})$, 2.41-2.05 (m, $2 \mathrm{H}), 1.85(\mathrm{~s}, 3 \mathrm{H}), 1.53(\mathrm{~s}, 3 \mathrm{H}), 1.31(\mathrm{~s}, 3 \mathrm{H}) .{ }^{13} \mathrm{C}-\mathrm{NMR}\left(\mathrm{CDCl}_{3}, 75 \mathrm{MHz}\right): \delta(\mathrm{ppm})$ 170.27 (CO), 138.57, 138.45, 138.37, 137.50, 128.40, 128.38, 128.36, 128.29, 128.14, 127.99, 127.83, $127.74,127.63,127.58,127.43,117.27,113.08,104.17,79.81,78.99,77.20,76.46,75.53,75.22$, 73.93, 73.57, 73.97, 73.57, 73.28, 72.84, 72.21, 71.92, 71.75, 69.28, 68.92, 34.43, 26.80, 26.60, 21.00. $[\alpha]_{\mathrm{D}}^{25}+43.73^{\circ}\left(c \quad 1.65\right.$ in $\left.\mathrm{CHCl}_{3}\right)$. HR-MS (ESI), $m / z, 831.37633[\mathrm{M}+\mathrm{Na}]^{+}\left(\mathrm{C}_{48} \mathrm{H}_{56} \mathrm{NaO}_{11}{ }^{+}\right.$, requires 831.37148).

2-[3-O-(5-O-Acetyl-3-O-benzyl-1,2-O-isopropylidene-6-deoxy- $\alpha$-D-allofuranos-6-yl)-2,4,6-tri-O-benzyl$\alpha$-D-mannopyranosyl]acetaldehyde (17). To a mixture of alkene $16(0.850 \mathrm{~g}, 1.05 \mathrm{mmol})$ and $\mathrm{N}$-methylmorpholine $\mathrm{N}$-oxide (NMO) $(213 \mathrm{mg}, 1.58 \mathrm{mmol})$ in acetone $(6 \mathrm{~mL})$ and water $(2 \mathrm{~mL})$ was 
added osmium tetroxide $(0.2 \mathrm{w} \%$ in water, $2.6 \mathrm{~mL}, 0.021 \mathrm{mmol}, 0.02$ eq. $)$ at $0{ }^{\circ} \mathrm{C}$. The resulting mixture was stirred for $45 \mathrm{~h}$ when TLC revealed the disappearance of the alkene 16. Upon addition of sodium sulfite $(1.12 \mathrm{~g})$ the yellow suspension turned into a slightly yellow two-layered solution. The upper organic layer was separated, the aqueous layer was extracted with EtOAc, the combined organic layers were dried $\left(\mathrm{MgSO}_{4}\right)$ and concentrated to give the crude diol $(802 \mathrm{mg}, 91 \%)$ as a mixture of two epimers in a ratio of ca. 0.75:1 (based on the integrals in the ${ }^{1} \mathrm{H}-\mathrm{NMR}$ spectrum). IR $\left(\mathrm{cm}^{-1}, \mathrm{CHCl}_{3}\right)$ : 3473 (w), 3031 (vw), 2931 (w), 2871 (w), 1737 (m), 1496 (w), 1454 (m), 1372 (m), 1236 (s), 1164 (w), 1090 (s), 1070 (s), 1024 (s). ${ }^{1} \mathrm{H}-\mathrm{NMR}\left(\mathrm{CDCl}_{3}, 300 \mathrm{MHz}\right): \delta(\mathrm{ppm}) 7.32-7.24$ (m, $\left.32.3 \mathrm{H}\right), 5.63$ (d, $0.6 \mathrm{H}, J=3.0 \mathrm{~Hz}), 5.60(\mathrm{~d}, 0.8 \mathrm{H}, J=3.3 \mathrm{~Hz}), 5.29-5.23(\mathrm{~m}, 1.5 \mathrm{H}), 5.67-4.39(\mathrm{~m}, 13.9 \mathrm{H}), 4.17-4.06$ $(\mathrm{m}, 4.0 \mathrm{H}), 3.95-3.78(\mathrm{~m}, 7.2 \mathrm{H}), 3.72-3.42(\mathrm{~m}, 13.9 \mathrm{H}), 4.17-4.06(\mathrm{~m}, 4.0 \mathrm{H}), 3.95-3.78(\mathrm{~m}, 7.2 \mathrm{H})$, 3.72-3.42 (m, $13.9 \mathrm{H}), 2.04(\mathrm{~d}, 1.2 \mathrm{H}, J=0.9 \mathrm{~Hz}), 1.84-1.83(\mathrm{~m}, 5.1 \mathrm{H}), 1.78-1.51(\mathrm{~m}, 2.9 \mathrm{H}), 1.52$ $(\mathrm{s}, 5.3 \mathrm{H}), 1.31$ (br s, $5.2 \mathrm{H}) .{ }^{13} \mathrm{C}-\mathrm{NMR}\left(\mathrm{CDCl}_{3}, 75 \mathrm{MHz}\right): \delta(\mathrm{ppm}) 170.29,138.17,138.10,138.07$, $138.00,137.98,137.49,137.46,128.46,128.42,128.16,128.14,128.02,127.94,127.90,127.83$, $127.80,127.74,127.70,127.66,133.11,133.09,104.15,79.84,77.20,76.79,76.67,76.40,76.31$, 75.25, 73.61, 73.45, 73.42, 73.18, 71.93, 71.80, 69.47, 69.39, 66.38, 32.81, 32.69, 26.82, 26.60, 21.01. HR-MS(ESI), $m / z, 865.37635[\mathrm{M}+\mathrm{Na}]^{+}\left(\mathrm{C}_{48} \mathrm{H}_{58} \mathrm{NaO}_{13}{ }^{+}\right.$, required 865.37696).

To the diol $(630 \mathrm{mg}, 0.747 \mathrm{mmol})$ in $\mathrm{MeOH}(3 \mathrm{~mL})$ and $\mathrm{H}_{2} \mathrm{O}(5 \mathrm{~mL})$ was added sodium periodate (190 mg, $0.897 \mathrm{mmol})$ in $\mathrm{H}_{2} \mathrm{O}(3 \mathrm{~mL})$ at $0{ }^{\circ} \mathrm{C}$, the mixture was stirred for $60 \mathrm{~min}$, and then extracted with EtOAc. The combined organic layers were dried $\left(\mathrm{Mg}_{2} \mathrm{SO}_{4}\right)$ and concentrated to give the aldehyde 17 as a slightly yellow oil which was pure according to the NMR spectrum. IR $\left(\mathrm{cm}^{-1}, \mathrm{CHCl}_{3}\right): 3030$ (vw), 2933 (w), 2870 (w), 1739 (m), 1726 (m), 1496 (w), 1454 (w), 1371 (m), 1307 (m), 1234 (s), 1091 (s), 1071 (s), 1025 (s). ${ }^{1} \mathrm{H}-\mathrm{NMR}\left(\mathrm{CDCl}_{3}, 300 \mathrm{MHz}\right): \delta$ (ppm) 9.68 (t, $\left.1 \mathrm{H}, J=2.3 \mathrm{~Hz}\right), 7.36-7.21$ $(\mathrm{m}, 20 \mathrm{H}), 5.63(\mathrm{~d}, 1 \mathrm{H}, J=3.7 \mathrm{~Hz}), 5.28(\mathrm{ddd}, 1 \mathrm{H}, J=4.6,3.4,6.4 \mathrm{~Hz}), 4.74-4.42(\mathrm{~m}, 10 \mathrm{H}), 4.13$ (dd, $1 \mathrm{H}, J=5.2,8.9 \mathrm{~Hz}), 3.96-3.61(\mathrm{~m}, 9 \mathrm{H}), 2.62(\mathrm{dd}, 1 \mathrm{H}, J=1.1,2.0 \mathrm{~Hz}), 2.60(\mathrm{t}, 1 \mathrm{H}, J=2.3 \mathrm{~Hz})$, $1.87(\mathrm{~s}, 3 \mathrm{H}), 1.55(\mathrm{~s}, 3 \mathrm{H}), 1.34(\mathrm{~s}, 3 \mathrm{H}) .{ }^{13} \mathrm{C}-\mathrm{NMR}\left(\mathrm{CDCl}_{3}, 75 \mathrm{MHz}\right): \delta(\mathrm{ppm}) 200.50,170.21$, $138.40,138.07,137.92,137.79,137.54,129.09,128.50,128.48,128.44,128.38,128.11,128.08$, $128.03,127.90,127.87,127.82,127.79,127.57,125.36,113.11,104.17,79.80,77.21,76.52,76.43$, $76.00,74.91,74.43,73.24,72.85,72.18,71.80,71.49,69.65,68.36,66.57,45.32,26.53,26.62,21.52$, 21.00. $[\alpha]_{\mathrm{D}}^{25}+56.27^{\circ}$ (c 2.00 in $\mathrm{CHCl}_{3}$ ). HR-MS(ESI), $m / z, 833.35133[\mathrm{M}+\mathrm{Na}]^{+}\left(\mathrm{C}_{47} \mathrm{H}_{54} \mathrm{NaO}_{12}{ }^{+}\right.$, required 833.35075$)$.

2-[3-O-(5-O-Acetyl-3-O-benzyl-1,2-O-isopropylidene-6-deoxy- $\alpha$-D-allofuranos-6-yl)-2,4,6-tri-O-benzyl$\alpha$-D-mannopyranosyl]acetic acid (18). The crude aldehyde 17 in acetonitrile $(6 \mathrm{~mL})$ was treated with $\mathrm{NaH}_{2} \mathrm{PO}_{4}(34 \mathrm{mg}, 0.25 \mathrm{mmol})$ in $\mathrm{H}_{2} \mathrm{O}(2 \mathrm{~mL})$ and $\mathrm{H}_{2} \mathrm{O}_{2}(30 \%, 0.12 \mathrm{~mL}, 1.12 \mathrm{mmol})$, respectively, at $0{ }^{\circ} \mathrm{C}$, followed by addition $\mathrm{NaClO}_{2}(0.17 \mathrm{~g}, 80 \%, 1.49 \mathrm{mmol})$ in $\mathrm{H}_{2} \mathrm{O}(2 \mathrm{~mL})$ at $0{ }^{\circ} \mathrm{C}$. The resulting mixture was stirred overnight, brought to $\mathrm{pH} 2.0$ with $1 \mathrm{~N} \mathrm{HCl}$, and extracted with EtOAc. The combined organic layers were dried $\left(\mathrm{MgSO}_{4}\right)$ and concentrated to give acid $\mathbf{1 8}$ as a colorless gum (0.60 g, 88\% from 16). IR( $\left.\mathrm{cm}^{-1}, \mathrm{CHCl}_{3}\right): 3062(\mathrm{w}), 3030(\mathrm{w}), 2933(\mathrm{w}), 2872(\mathrm{w}), 1739$ (s), $1714(\mathrm{~m})$, 1496 (w), 1454 (w), 1372 (m), 1306 (w), 1236 (s), 1162 (m), 1095 (s), 1026 (s). ${ }^{1} \mathrm{H}-\mathrm{NMR}\left(\mathrm{CDCl}_{3}\right.$, $300 \mathrm{MHz}): \delta(\mathrm{ppm}) 7.33-7.25(\mathrm{~m}, 20 \mathrm{H}), 5.61(\mathrm{~d}, 1 \mathrm{H}, J=3.6 \mathrm{~Hz}), 5.23(\mathrm{dd}, 1 \mathrm{H}, J=5.3,10.2 \mathrm{~Hz})$, 4.68-4.36 (m, $10 \mathrm{H}), 4.34-4.30(\mathrm{~m}, 1 \mathrm{H}), 4.10(\mathrm{dd}, 1 \mathrm{H}, J=5.3,8.9 \mathrm{~Hz}), 3.97$ (br. s, $1 \mathrm{H}), 3.89-3.78$ $(\mathrm{m}, 3 \mathrm{H}), 3.73(\mathrm{~d}, 1 \mathrm{H}, J=2.2 \mathrm{~Hz}), 3.64-3.56(\mathrm{~m}, 3 \mathrm{H}), 2.70(\mathrm{dd}, 1 \mathrm{H}, J=4.3,15.7 \mathrm{~Hz}), 2.56(\mathrm{dd}$, 
$1 \mathrm{H}, J=8.6,15.9 \mathrm{~Hz}), 1.84(\mathrm{~s}, 3 \mathrm{H}), 1.52(\mathrm{~s}, 3 \mathrm{H}), 1.31(\mathrm{~s}, 3 \mathrm{H}) .{ }^{13} \mathrm{C}-\mathrm{NMR}\left(\mathrm{CDCl}_{3}, 75 \mathrm{MHz}\right): \delta(\mathrm{ppm})$ $175.43,170.35,138.31,138.06,137.88,137.50,128.47,128.43,128.36,128.17,128.04,127.97$, $127.89,127.86,127.81,127.56,133.13,104.17,79.80,77.20,76.86,76.45,75.66,74.84,74.47,73.33$, $73.05,72.24,71.84,71.50,69.45,68.40,68.16,36.25,26.98,26.81,26.61,20.98 .[\alpha]_{\mathrm{D}}^{25}+50.97^{\circ}(c 4.52$ in $\left.\mathrm{CHCl}_{3}\right)$. HR-MS(ESI), $m / z, 849.34625[\mathrm{M}+\mathrm{Na}]^{+}\left(\mathrm{C}_{47} \mathrm{H}_{54} \mathrm{NaO}_{13}{ }^{+}\right.$, requires 849.34621), 871.32809 $\left[\mathrm{M}-\mathrm{H}+\mathrm{Na}_{2}\right]^{+}\left(\mathrm{C}_{47} \mathrm{H}_{534} \mathrm{Na}_{2} \mathrm{O}_{13}{ }^{+}\right.$, requires 871.32816).

2-[3-O-(5-O-Acetyl-3-O-benzyl-1,2-O-isopropylidene-6-deoxy- $\alpha$-D-allofuranos-6-yl)-2,4,6-tri-O-benzyl$\alpha$-D-mannopyranosyl]- $N$-[2-amino-6-(benzyloxy)-5-nitrosopyrimidin-4-yl]acetamide (19). To a solution of acid 18 (0.470 g, $0.57 \mathrm{mmol})$ in $\mathrm{CH}_{2} \mathrm{Cl}_{2}(4 \mathrm{~mL})$ was added oxalyl chloride $(0.15 \mathrm{~mL}, 1.7 \mathrm{mmol})$ followed by a drop of DMF at $0{ }^{\circ} \mathrm{C}$. The resulting mixture was stirred for $1 \mathrm{~h}$ and concentrated in vacuo. The crude acid chloride was pure according to its NMR spectra and used directly for next step. IR ( $\left.\mathrm{cm}^{-1}, \mathrm{CHCl}_{3}\right): 3031$ (vw), 2932 (vw), 2868 (vw), 1798 (m), 1740 (s), 1496 (w), 1454 (m), 1371 (m), 1234 (s), 1091 (s), 1069 (s), 1023 (s). ${ }^{1} \mathrm{H}-\mathrm{NMR}\left(\mathrm{CDCl}_{3}, 300 \mathrm{MHz}\right): \delta$ (ppm) 7.36-7.24 (m, $\left.20 \mathrm{H},\right)$, $5.61(\mathrm{~d}, 1 \mathrm{H}, J=3.7 \mathrm{~Hz}), 5.23(\mathrm{dd}, 1 \mathrm{H}, J=6.0,10.0 \mathrm{~Hz}), 4.95(\mathrm{~d}, 1 \mathrm{H}, J=11.0 \mathrm{~Hz}), 4.73-4.31(\mathrm{~m}, 9 \mathrm{H})$, $4.10(\mathrm{dd}, 1 \mathrm{H}, J=5.4,8.9 \mathrm{~Hz}), 3.97$ (t, $1 \mathrm{H}, J=5.8 \mathrm{~Hz}), 3.86$ (dd, $1 \mathrm{H}, J=4.3,8.8 \mathrm{~Hz}), 3.81-3.67$ (m, $4 \mathrm{H}), 3.64-3.57$ (m, 3 H), 3.21-3.14 (m, 1 H), 2.99-2.90 (m, $1 \mathrm{H}), 1.84$ (s, $3 \mathrm{H}), 1.52$ (s, $3 \mathrm{H}), 1.31$ (s, $3 \mathrm{H}) .{ }^{13} \mathrm{C}-\mathrm{NMR}\left(\mathrm{CDCl}_{3}, 75 \mathrm{MHz}\right): \delta$ (ppm) 171.27, 170.22, 138.39, 137.93, 137.54, 137.48, 128.55, $128.48,128.34,128.16,128.02,127.99,127.84,127.77,127.52,113.12,104.13,79.89,77.32,77.23$, $76.30,75.93,75.25,74.78,74.64,73.34,72.56,72.23,71.74,71.21,69.72,68.25,67.23,49.09,26.82$, 26.61, 20.95, 13.97. $[\alpha]_{\mathrm{D}}^{25}+43.68^{\circ}\left(c 2.35\right.$ in $\left.\mathrm{CHCl}_{3}\right)$.

To a solution of 2,4-diamino-5-nitrosopyrimidine $9(0.21 \mathrm{~g}, 0.86 \mathrm{mmol})$ and pyridine $(0.07 \mathrm{~mL}) \mathrm{in}$ dry THF $(12 \mathrm{~mL})$ was added the above acid chloride $(0.47 \mathrm{~g}, 0.57 \mathrm{mmol})$ in THF $(7 \mathrm{~mL})$ at $0{ }^{\circ} \mathrm{C}$ dropwise. Once the acid chloride was added, the deep-blue solution turned green. After stirring for $1 \mathrm{~h}$, the purple solid was filtered off, and the filtrate was concentrated in vacuo to afford the amide 19 (600 mg). IR ( $\left.\mathrm{cm}^{-1}, \mathrm{CHCl}_{3}\right)$ : 3328 (w), 3228 (w), 3031 (w), 2933 (w), 2870 (w), 1739 (m), 1631 (m), 1598 (s), 1535 (s), 1497 (m), 1454 (s), 1371 (m), 1347 (s), 1255 (s), 1209 (s), 1091 (s), 1072 (s), 1026 (s). ${ }^{1} \mathrm{H}-\mathrm{NMR}\left(\mathrm{CDCl}_{3}, 300 \mathrm{MHz}\right): \delta$ (ppm) 12.38 (br. s, $1 \mathrm{H}$, exchange with $\left.\mathrm{D}_{2} \mathrm{O}\right), 7.51-7.23(\mathrm{~m}, 20 \mathrm{H}), 7.17$ (br. s, $1 \mathrm{H}$, exchange with $\mathrm{D}_{2} \mathrm{O}$ ), 6.03 (br. s, $1 \mathrm{H}$, exchange with $\left.\mathrm{D}_{2} \mathrm{O}\right), 5.65(\mathrm{~d}, 1 \mathrm{H}, J=3.7 \mathrm{~Hz}), 5.63$ $(\mathrm{s}, 2 \mathrm{H}), 5.27(\mathrm{dd}, 1 \mathrm{H}, J=5.8,10.0 \mathrm{~Hz}), 4.70(\mathrm{~d}, 1 \mathrm{H}, J=11.0 \mathrm{~Hz}), 4.64(\mathrm{~d}, 1 \mathrm{H}, J=11.6 \mathrm{~Hz}), 4.63$ (d, $1 \mathrm{H}, J=11.8 \mathrm{~Hz}), 4.57-4.43(\mathrm{~m}, 6 \mathrm{H}), 4.14(\mathrm{dd}, 1 \mathrm{H}, J=5.5,8.9 \mathrm{~Hz}), 4.06$ (br. s, $1 \mathrm{H}), 3.88$ (dd, $1 \mathrm{H}$, $J=4.3,8.9 \mathrm{~Hz}), 3.83-3.65(\mathrm{~m}, 7 \mathrm{H}), 2.97(\mathrm{dd}, 1 \mathrm{H}, J=5.3,14.7 \mathrm{~Hz}), 2.89$ (dd, $1 \mathrm{H}, J=7.6,14.9 \mathrm{~Hz})$, $1.83(\mathrm{~s}, 3 \mathrm{H}), 1.51(\mathrm{~s}, 3 \mathrm{H}), 1.32(\mathrm{~s}, 3 \mathrm{H}) .{ }^{13} \mathrm{C}-\mathrm{NMR}\left(\mathrm{CDCl}_{3}, 75 \mathrm{MHz}\right): \delta(\mathrm{ppm}) 171.45,170.35$, $163.78,138.91,138.31,138.04,137.86,137.46,135.49,128.64,128.46,128.44,128.32,128.26$, $128.20,128.06,127.95,127.80,127.72,127.51,113.12,104.15,79.92,77.33,77.24,76.20,76.05$, $74.74,74.12,73.13,72.25,71.91,71.62,69.40,68.72,68.62,41.33,26.72,26.61,20.97 .[\alpha]_{\mathrm{D}}^{25}+81.92^{\circ}$ (c 0.053 in $\left.\mathrm{CHCl}_{3}\right)$. HR-MS (ESI), $m / z, 1076.4270[\mathrm{M}+\mathrm{Na}]^{+}\left(\mathrm{C}_{58} \mathrm{H}_{63} \mathrm{~N}_{5} \mathrm{NaO}_{14}{ }^{+}\right.$, required 1076.4264).

8-[(3-O-((5-O-Acetyl-3-O-benzyl-1,2-O-isopropylidene-6-deoxy- $\alpha$-D-allofuranos-6-yl)-2,4,6-tri-O-benzyl$\alpha$-D-mannopyranosyl)methyl]- $O^{6}$-benzylguanine (6). A mixture of the amide 19 (600 $\left.\mathrm{mg}, 0.57 \mathrm{mmol}\right)$ and triphenyl phosphine $(449 \mathrm{mg}, 1.71 \mathrm{mmol})$ in xylene was stirred at $140{ }^{\circ} \mathrm{C}$ overnight and then cooled to room temperature. The mixture was passed through silica gel $($ EtOAc/cyclohexane $1 / 1 \rightarrow 1 / 0)$ 
to give guanine 6 as a yellowish oil $\left(291 \mathrm{mg}, 50 \%\right.$ from acid 18). IR ( $\left.\mathrm{cm}^{-1}, \mathrm{CHCl}_{3}\right): 3528(\mathrm{w}), 3420$ (w), 3067 (w), 3010 (m), 2928 (m), 1738 (m), 1625 (s), 1591 (s), 1496 (w), 1455 (m), 1374 (m), 1325 (m), 1227 (s), 1146 (m), 1090 (s). ${ }^{1} \mathrm{H}-\mathrm{NMR}\left(\mathrm{CDCl}_{3}, 300 \mathrm{MHz}\right): \delta$ (ppm) 11.65 (br s, $1 \mathrm{H}, \mathrm{NH}$, exchange with $\left.\mathrm{D}_{2} \mathrm{O}\right), 7.50-7.20(\mathrm{~m}, 25 \mathrm{H}), 5.57(\mathrm{~d}, 1 \mathrm{H}, J=3.7 \mathrm{~Hz}), 5.53(\mathrm{~s}, 2 \mathrm{H}), 5.18(\mathrm{dd}, 1 \mathrm{H}$, $J=5.0,10.7 \mathrm{~Hz}), 4.80$ (br. s, $2 \mathrm{H}$, exchange with $\left.\mathrm{D}_{2} \mathrm{O}\right), 4.71-4.35(\mathrm{~m}, 10 \mathrm{H}), 4.21$ (t, $1 \mathrm{H}, J=8.1 \mathrm{~Hz}$ ), $4.08(\mathrm{dd}, 2 \mathrm{H}, J=5.0,8.9 \mathrm{~Hz}), 3.86-3.70(\mathrm{~m}, 3 \mathrm{H}), 3.61(\mathrm{dd}, 1 \mathrm{H}, J=2.6,7.7 \mathrm{~Hz}), 3.58-3.53$ (m, $2 \mathrm{H}), 3.47$ (dd, $1 \mathrm{H}, J=4.0,10.2 \mathrm{~Hz}), 3.30$ (d, $1 \mathrm{H}, J=14.0 \mathrm{~Hz}), 2.97$ (dd, $1 \mathrm{H}, J=10.8,16.0 \mathrm{~Hz}$ ), $1.84(\mathrm{~s}, 3 \mathrm{H}), 1.49(\mathrm{~s}, 3 \mathrm{H}), 1.30(\mathrm{~s}, 3 \mathrm{H}) .{ }^{13} \mathrm{C}-\mathrm{NMR}\left(\mathrm{CDCl}_{3}, 75 \mathrm{MHz}\right): \delta(\mathrm{ppm}) 170.14,160.10$, $158.72,155.66,148.61,137.74,137.65,137.42,136.79,132.20,132.07,128.61,128.56,128.49$, $128.46,128.40,128.35,128.32,128.22,128.04,127.98,127.92,127.82,127.69,114.66,13.06$, 104.16, 79.68, 77.26, 77.11, 76.47, 75.95, 75.27, $74.37\left(\mathrm{CH}_{2}\right), 73.14,72.70,72.15,71.79,69.86$, 67.79, 26.95, 26.78, 26.55, 20.98. $[\alpha]_{\mathrm{D}}^{25}+31.76^{\circ}$ (c 1.41 in $\mathrm{CHCl}_{3}$ ). HR-MS (ESI), $m / z, 1044.4378$ $[\mathrm{M}+\mathrm{Na}]^{+}\left(\mathrm{C}_{58} \mathrm{H}_{63} \mathrm{~N}_{5} \mathrm{NaO}_{12}{ }^{+}\right.$, required 1044.4365).

\section{Conclusions}

In summary, a new linear analogue of guanofosfocin $\mathbf{6}$ was synthesized in 10 steps from the known sugar diol 13 in $26 \%$ overall yield. Key steps were the ring-opening of cyclic sulfate $\mathbf{8}$ by a sugar sec-alkoxide and the reductive cyclization of 4- $\mathrm{N}$-acyl-2,4-diamino-5-nitrosopyrimidine 19. The robustness of the above etherification and of the reductive cyclization will find more applications in synthetic organic chemistry. The structure of $\mathbf{6}$ was characterized as an ether-linked pseudodisaccharide $\alpha$ - $C$-glycosidically linked to an 8-alkylated guanine. This compound, together with previously synthesized analogues of guanofosfocins, allows expansion of the relevant research in this field of medicinal chemistry and chemical biology.

\section{Acknowledgments}

We thank ETH Zürich and The National Natural Science Foundation of China (No. 21202008) for generous support.

\section{References}

1. Varki, A. Biological roles of oligosaccharides: All of the theories are correct. Glycobiology 1993, 3, 97-130.

2. Chapleur, Y. Carbohydrate Mimics; Wiley: New York, NY, USA, 1998.

3. Lindhorst, T.K. Glycomimetics: Tools for investigation of functional diversity in the carbohydrate regime. Proceedings of the International Beilstein Symposium on Glyco-Bioinformatics; Hicks, M.G., Kettner, C., Eds.; Logos-Verlag Berlin: Berlin, Germany, 2011; pp. 147-164.

4. Nicotra, F. Synthesis of $C$-glycosides of biological interest. In Topics in Current Chemistry; Driguez, H., Thiem, J., Eds.; Springer-Verlag: Berlin, Germany, 1997; pp. 55-83.

5. Sardinha, J.; Guieu, S.; Deleuze, A.; Fernández-Alonso, M. C.; Rauter, A. P.; Sina, P.; Marrot, J.; Jiménez-Barbero, J.; Sollogoub, M. gem-Difluoro-carbasugars, the cases of mannopyranose and galactopyranose. Carbohydr. Res. 2007, 342, 1689-1703. 
6. Driguez, H. Thiooligosaccharides in glycobiology. In Topics in Current Chemistry; Driguez, H., Thiem, J., Eds.; Springer-Verlag: Berlin, Germany, 1997; pp. 85-116.

7. Katoh, H.; Yamada, M.; Lida, K.; Aoki, M.; Itezono, Y.; Nakayama, N.; Suzuki, Y.; Watanabe, M.; Shimada, H. Fujimari, H.; et al. Isolation and structure elucidation of novel Chitin synthase inhibitors, guanofosfocins produced by microorganisms. Tennen Yuki Kagobutsu Toronkai Koen Yoshishu 1996, 38, 115-120.

8. Sugimura, H.; Stansfield, K. Synthesis of 8-(mannosyloxy)adenosine: A novel nucleosidecarbohydrate hybrid. Synlett 1998, 1998, 985-986.

9. Stansfield, K.; Kanamori, H.; Sugimura, H. Synthesis of novel nucleoside.carbohydrate hybrids. New J. Chem. 1999, 23, 9-11.

10. Kiyohara, W.; Natsui, Y.; Koizumi, A.; Sugimura, H. Synthetic study of guanofosfocin. Synthesis of 8-(mannopyranosyloxy)inosine derivative. Nucleic Acids Res. Suppl. 2001, 2001, 183-184.

11. Sugimura, H.; Hosogai, N. Studies toward stable analogues of guanofosfocins. Synthesis of the protected derivative of 8-(5a-carba- $\alpha$-D-mannopyranosyloxy)purine nucleoside. Chem. Lett. 2007, $36,36-37$.

12. George, T.G.; Szolcsányi, P.; Koenig, S.G.; Paterson, D.E.; Isshiki, Y.; Vasella, A. Preparation of an advanced intermediate for the synthesis of stable analogues of guanofosfocin. Helv. Chim. Acta 2004, 87, 1287-1298.

13. Duchek, J.; Huang, M.-H.; Vasella, A. Towards stable di-carba analogues of guanofosfocins. Tetrahedron Lett. 2011, 52, 2940-2952.

14. Akhtar, T.; Eriksson, L.; Cumpstey, I. Unusual synthesis of carbohydrate sec-sec ether-linked pseudodisaccharides. Carbohydr. Res. 2008, 343, 2094-2100.

15. Takahashi, H.; Fukuda, T.; Mitsuzuka, H.; Namme, R.; Miyamoto, H.; Ohkura, Y.; Ikegami, S. Development of a novel sugar linkage: 6,6'-Ether-connected sugars. Angew. Chem. Int. Ed. Engl. 2003, 42, 5069-5071.

16. Haines, A.H. Synthesis of 6,6'-ether linked disaccharides from D-allose, D-galactose, D-glucose and D-mannose; evidence on the structure of coyolosa. Org. Biomol. Chem. 2004, 2, 2352-2358.

17. Gourlain, T.; Wadouachi, A.; Uzan, R.; Beaupère, D. Synthesis of ether linked pseudo-oligosaccharides via 5,6-cyclic sulfate derivatives of protected manno and glucofuranose. J. Carbohydr. Chem. 1997, 16, 1089-1100.

18. Xu, M.; de Giacomo, F.; Paterson, D.E.; George, T.G.; Vasella, A. An improved procedure for the preparation of 8-substituted guanines. Chem. Commun. 2003, 2003, 1452-1453.

19. Glen, W.L.; Myers, G.S.; Grant, G.A. Monoalkyl hexoses-Improved procedures for the preparation of 1-methyl and 3-methyl ethers of fructose, and of 3-alkyl ethers of glucose. J. Chem. Soc. 1951, 1951, 2568-2572.

20. Loiseleur, O.; Ritson, D.; Nina, M.; Crowley, P.; Wagner, T.; Hanessian, S. Ring-modified analogues and molecular dynamics studies to probe the requirements for fungicidal activities of malayamycin a and its N-nucleoside variants. J. Org. Chem. 2007, 72, 6353-6363.

21. Yoshikawa, M.; Okaichi, Y.; Cha, B.C.; Kitagawa, I. Synthesis of (-)-aristeromycin from D-glucose. Tetrahedron 1990, 46, 7459-7470. 
22. Nacro, K.; Lee, J.; Barchi, J.J.; Lewin, N.E.; Blumberg, P.M.; Marquez, V.E. Conformationally constrained analogues of diacylglycerol (DAG). Part 19: Asymmetric syntheses of (3R)- and (3S)-3-hydroxy-4,4-disubstituted heptono-1,4-lactones as protein kinase C (PK-C) ligands with increased hydrophilicity. Tetrahedron 2002, 58, 5335-5345.

23. Hanaya, T.; Sugiyama, K.; Kawamoto, H.; Yamamoto, H. Stereoselectivity in deoxygenation of 5-hydroxy-5-phosphinyl-hexofuranoses (alpha-hydroxyphosphonates). Carbohydr. Res. 2003, 338, 1641-1650.

24. Gao, Y.; Sharpless, K.B. Vicinal diol cyclic sulfates-like epoxides only more reactive. J. Am. Chem. Soc. 1988, 110, 7538-7539.

Sample Availability: Not available.

(C) 2013 by the authors; licensee MDPI, Basel, Switzerland. This article is an open access article distributed under the terms and conditions of the Creative Commons Attribution license (http://creativecommons.org/licenses/by/3.0/). 